Nordic Machine Intelligence, MedAI 2021

https://doi.org/10.5617/nmi.9171

\title{
Transformer Based Multi-model Fusion for Medical Image Segmentation
}

\author{
Bo Dong ${ }^{1}$, Wenhai Wang ${ }^{2 *}$ and Jinpeng $\mathrm{Li}^{3}$ \\ 1. College of Biomedical Engineering \& Instrument Science, Zhejiang University, Zhejiang, China. \\ 2. Department of Computer Science and Technology, Nanjing University, Nanjing, China. \\ 3. Inception Institute of Artificial Intelligence, Abu Dhabi, UAE. \\ Corresponding authors: Wenhai Wang (wangwenhai362@smail.nju.edu.cn)
}

\begin{abstract}
We present our solutions to the MedAl for all the three tasks: polyp segmentation task, instrument segmentation task, and transparency task. We use the same framework to process the two segmentation tasks of polyps and instrument. The key improvement over last year is new state-of-the-art vision architectures, especially transformers which significantly outperform ConvNets for the medical image segmentation tasks. Our solution consists of multiple segmentation models, and each model uses a transformer as the backbone network. we get the best loU score of 0.915 on instrument segmentation task and 0.836 on polyp segmentation task after submitting. Meanwhile, we provide complete solutions in https://github. com/dongbo811/MedAl-2021.
\end{abstract}

Keywords: polyp segmentation; surgical instrument segmentation; vision transformer

\section{Introduction \\ Colorectal cancer is one of the deadliest cancers in the world. Colonoscopy is the standard method for locating and removing colorectal polyps. However, there is a high missed diagnosis rate for polyps. Therefore, an automatic and accurate polyp segmentation [1] method during colonoscopy can help clinicians eliminate missed lesions and prevent the development of colorectal cancer. Meanwhile, surgical instruments [1] can be used for robot-assisted surgical tasks to reduce surgical risks and workload of doctors, which has important clinical significance.}

\section{Materials and Methods}

Following the official regulations, we train our models on the Kvasir-seg [2] with 1000 images and Kvasirinstrument [3] containing 590 images to complete the polyp segmentation and instrument segmentation tasks, respectively. Our method is the ensemble of three submodels, namely Polyp-PVT, Sinv2-PVT, and TransfusePVT. The official Polyp-PVT [4] is designed for polyp segmentation and achieves SOTA segmentation capability and generalization performance. Here, we adopt its standard structure without any modification. For the Transfuse [5], which is also designed for polyp segmentation, we improve it by replacing the transformer part with the pyramid vision transformer (PVT) $[6,7]$ to enhance its performance. The official Sinv2 [8] proposes an end-toend network for searching and recognizing the concealed object, which achieves considerable segmentation performance. Due to the similarity of segmentation tasks, we employ it and replace the original backbone of Res2Net [9] with a stronger PVT transformer $[6,7]$ to extract more powerful features.

\section{Hyperparameter Settings}

We use Adam optimizer to optimize our models with the initial learning rate of $1 \mathrm{e}-4$. In addition, we resize the input image to $352 \times 352$, and set the batch size to 16 . To adapt to the images with different sizes, we use multiscale strategy in the training phase.

\section{Training Stage}

We use the same training strategy to train Polyp-PVT, Sinv2-PVT, and Transfuse-PVT. Specifically, we first divide the dataset into 5 groups (folds). At each time, we use 4 groups of dataset as the training set and the remaining one as the validation set. During training, we choose the model weights with the best loU on the validation set. After 5-fold training, each of Polyp-PVT, Sinv2-PVT, and Transfuse-PVT has 5 model weights, and a total of 15 different model weights are obtained. 
Table 1: 5-fold cross-validation results of surgical instrument segmentation and polyp segmentation. Intersection-OverUnion (Jaccard index), Dice coefficient, and the pixel accuracy are ab breviated as lo U, Dice, PA re spectively. The best mean score of each metrics in the three methods are marked in bold. The last line is the best score we got after submitting.

\begin{tabular}{|c|c|c|c|c|c|c|c|c|c|c|c|c|c|}
\hline \multirow[b]{2}{*}{ models } & \multirow[b]{2}{*}{ Metrics } & \multicolumn{6}{|c|}{ Surgical Instrument Segmentation } & \multicolumn{6}{|c|}{ Polyp Segmentation } \\
\hline & & |Flod-1 & Flod-2 & Flod-3 & Flod-4 & Flod-5 & Mean & Flod-1 & Flod-2 & Flod-3 & Flod-4 & Flod-5 & Mean \\
\hline \multirow{3}{*}{ Polyp-PVT } & $\mathrm{loU}$ & 0.901 & 0.874 & 0.931 & 0.953 & 0.952 & 0.922 & 0.842 & 0.846 & 0.863 & 0.863 & 0.851 & 0.853 \\
\hline & Dice & 0.943 & 0.923 & 0.962 & 0.975 & 0.975 & 0.955 & 0.902 & 0.905 & 0.917 & 0.921 & 0.908 & 0.911 \\
\hline & PA & 0.992 & 0.990 & 0.994 & 0.994 & 0.993 & 0.993 & 0.967 & 0.968 & 0.974 & 0.973 & 0.969 & 0.970 \\
\hline \multirow{3}{*}{ Sinv2-PVT } & loU & 0.897 & 0.876 & 0.931 & 0.952 & 0.952 & 0.922 & 0.850 & 0.849 & 0.867 & 0.865 & 0.857 & 0.858 \\
\hline & Dice & 0.939 & 0.924 & 0.962 & 0.975 & 0.975 & 0.955 & 0.908 & 0.909 & 0.921 & 0.922 & 0.912 & 0.914 \\
\hline & PA & 0.991 & 0.990 & 0.994 & 0.994 & 0.993 & 0.992 & 0.970 & 0.967 & 0.974 & 0.972 & 0.971 & 0.971 \\
\hline \multirow{3}{*}{ Transfuse-PVT } & $\mathrm{loU}$ & 0.903 & 0.869 & 0.931 & 0.950 & 0.951 & 0.921 & 0.841 & 0.852 & 0.872 & 0.855 & 0.856 & 0.855 \\
\hline & Dice & 0.944 & 0.921 & 0.961 & 0.973 & 0.974 & 0.955 & 0.903 & 0.911 & 0.925 & 0.914 & 0.913 & 0.913 \\
\hline & PA & 0.992 & 0.989 & 0.993 & 0.993 & 0.993 & 0.992 & 0.967 & 0.968 & 0.977 & 0.970 & 0.969 & 0.970 \\
\hline \multicolumn{2}{|c|}{ Submission Score } & & .0 .9 & Dice & 50 & 0.989 & & & 50 & Dice: & 93 & PA: 0. & \\
\hline
\end{tabular}

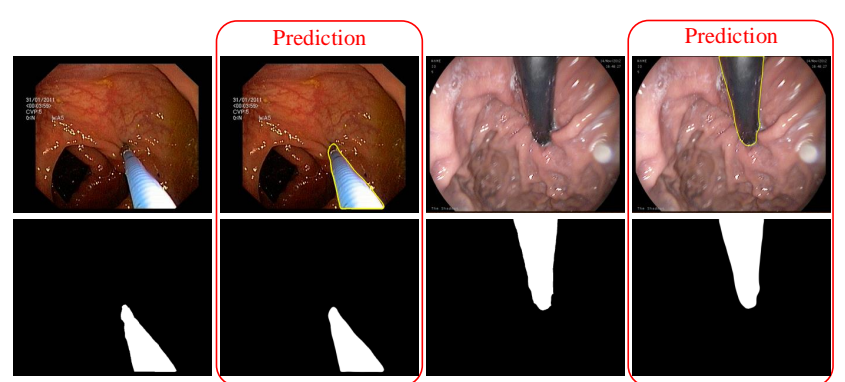

Figure 1: Qualitative description of our predication.

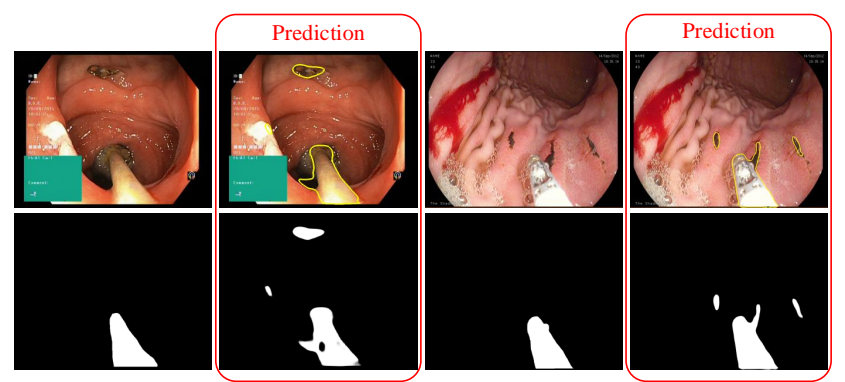

Figure 2: Some failure cases in surgical instrument segmentation.

\section{Inference Stage}

In the inference stage, we only resize the input images to $352 \times 352$ without any data enhancement. For the output, we upsample it to the original image size. So, we can obtain 15 different prediction results. In order to obtain a more robust prediction, we merge the 15 prediction results with a voting strategy where the minority obeys the majority. Because the voting strategy will produce many independent noise pixels on the edge of polyps or surgical instruments, we use the open operation and counting to remove tiny independent noise points. Then, the area of each block in the prediction image is counted to remove relatively small noise blocks in the prediction image.

\section{Result}

Due to space limitations, we only show the results of surgical instrument segmentation. We show the qualitative results in Figure 1, and give the results of evaluations in Table. 1. At the same time, we illustrate our failure cases in Figure 2.

\section{Discussion}

In the 5-fold cross-validation, the three results are relatively stable. There are results above 0.92 on the loU, and we also give some visual results in Figure 1 .

As shown in the Figure 1 and Table. 1, we have achieved great results in the surgical instrument segmentation, but there are still some failure cases as shown in Figure 2. Our results almost correctly segment the instruments, while introduce some noise. One characteristic of these noise is that they are biased towards black. This is due to the fact that there are many black instruments in the entire dataset. Therefore, small black areas (food residuals, etc.) in the colonoscope are identified as surgical instruments. These noises can be filtered by the size of the area to achieve the purpose of noise removal.

\section{Conclusion}

In this paper, we propose a robust generalized medical image segmentation framework, which is composed of multiple models, and uses the pyramid vision transformer backbone as an encoder to explicitly extract more powerful features. Meanwhile, for the transparency task, we provide a complete description of our method, data, open source code and results for easy reproduction.

\section{Acknowledgments}

I would first like to thank the researcher Dengping Fan in IIAI, whose expertise was invaluable in formulating the research questions and methodology. 


\section{References}

1. Hicks S, Jha D, Thambawita V, Riegler M, Halvorsen P, Singstad B, Gaur S, Pettersen K, Goodwin M, Parasa S, and Lange $T$ de. MedAl: Transparency in Medical Image Segmentation. Nordic Machine Intelligence 2021

2. Jha D, Smedsrud $P H$, Johansen $D$, Lange $T$ de, Johansen HD, Halvorsen $P$, and Riegler MA. A Comprehensive Study on Colorectal Polyp Segmentation with ResUNet++, Conditional Random Field and Test-Time Augmentation. 2020

3. Jha D, Ali S, Emanuelsen K, Hicks SA, Thambawita $V$, Garcia-Ceja E, Riegler MA, Lange T de, Schmidt PT, Johansen HD, Johansen D, and Halvorsen P. KvasirInstrument: Diagnostic and Therapeutic Tool Segmentation Dataset in Gastrointestinal Endoscopy. MultiMedia Modeling. $2021: 218-29$

4. Bo D, Wenhai W, Deng-Ping F, Jinpeng L, Huazhu F, and Ling S. Polyp-PVT: Polyp Segmentation with PyramidVision Transformers. 2021

5. Zhang $\mathrm{Y}$, Liu H, and Hu Q. Transfuse: Fusing transformers and cnns for medical image segmentation. 2021

6. Wang W, Xie E, Li X, Fan DP, Song K, Liang D, Lu T, Luo $P$, and Shao L. Pyramid Vision Transformer: A Versatile Backbone for Dense Prediction without Convolutions. IEEE ICCV. 2021

7. Wang W, Xie E, Li X, Fan DP, Song K, Liang D, Lu T, Luo $P$, and Shao L. Pvtv2: Improved baselines with pyramid vision transformer. arXiv preprint arXiv:2106.13797 2021

8. Fan DP, Ji GP, Cheng MM, and Shao L. Concealed Object Detection. IEEE TPAMI 2021

9. Gao S, Cheng MM, Zhao K, Zhang XY, Yang $M H$, and Torr PH. Res2net: A new multi-scale backbone architecture. IEEE transactions on pattern analysis and machine intelligence 2019 